Brain Dev Aug 2002;24:281-283). (Respond: Dr Tony Charman, Institute of Child Health, 30 Guildford Street, WC1N 1EH London, UK).

COMMENT. Signs of abnormal or delayed development (hypotonia and delayed motor milestones) are commonly observed in cases of Rett syndrome in the pre-regression period. Earliest and most frequent signs of regression are loss of hand use and communication skills. Early developmental history can aid in detection of risk factors for Rett syndrome, and before the onset of growth delay, gait ataxia, and hand stereopathies.

\title{
FAMILIAL INFANTILE BILATERAL STRIATAL NECROSIS
}

The clinical and radiological evolution of familial infantile bilateral striatal necrosis (IBSN) was evaluated in 11 of 15 affected children born to consanguineous Israeli Bedouin parents and reported from the Schneider Children's Medical Center, Petah Tikva and Sackler School of Medicine, Tel Aviv University, and other centers in Israel. Three were treated with oral biotin 100 $\mathrm{mg} /$ day. Inheritance was autosomal recessive. Untreated children showed signs of developmental arrest with onset at age 7 to 15 months, choreoathetosis and dysphagia, and a later onset of pendular nystagmus. MRI showed severe basal ganglia atrophy. Postmortem findings in one patient showed severe atrophy of lenticular nuclei with gliosis and neuronal loss. Biotin therapy resulted in arrest or improvement of disease in 2 patients when administered early, and slowed progression in the proband with treatment over a 15 month period. (Straussberg $\mathrm{R}$, Shorer Z, Weitz R et al. Familial infantile bilateral striatal necrosis. Clinical features and response to biotin treatment. Neurology October ( 1 of 2) 2002;59:983989). (Reprints: Dr Rachel Straussberg, Neurogenetic Clinic, Department of Neurology, Schneider Children's Medical Center of Israel, Petah Tikva, Israel 49202).

COMMENT. Infantile bilateral striatal necrosis (IBSN) is a rare clinically heterogeneous syndrome characterized pathologically by symmetric spongy degeneration of the caudate nucleus, putamen, and occasionally the globus pallidus. Clinical manifestations are developmental regression, choreoathetosis, dystonia, dysphagia, and mental retardation. Prognosis is usually poor with spastic quadriparesis and early morbidity. Reported cases have been described in 3 groups: 1) subacute necrotizing encephalomyelopathy (Leigh disease); 2) familial striatal degeneration with slow progression; and 3) abrupt neurologic onset following an acute systemic illness. The Israeli familial cases described above are in group 2, with poor prognosis. Biotin is worthy of trial and early treatment is recommended.

\section{NOVEL ACTIN AND COFILIN AGGREGATIONS IN JUVENILE- ONSET DYSTONIA}

The brains of identical twins with juvenile-onset dystonia were examined at Emory University, Atlanta, Georgia. Clinically, the twins had only a mild developmental delay until age 12 years, and they then showed a rapidly progressive generalized dystonia and dementia, with death occurring at ages 21 and 22 years. Clinical findings and course were distinct from primary and secondary dystonias previously described. The twins were born with cleft lip and palate, their limbs were small, and skeletal abnormalities included high foreheads, hypoplastic scapulas, and kyphoscoliosis by age 10 years. Achalasia was diagnosed at age 2, cataracts at age 3, and sensory-neural deafness at age 4 . Dystonia developed first in the leg by age 14, and progressed over 5 years from a clumsy gait to an inability to walk. Oculogyric and opisthotonic crises occurred as 